\title{
Detecting land cover change using Sentinel-2
}

\author{
Koji Osumi $^{\mathrm{a}}$ \\ ${ }^{a}$ Geospatial Information Authority of Japan, Tsukuba, Ibaraki, Japan; osumi-k96mz@mlit.go.jp
}

Keywords: Sentinel-2, land cover change, normalized difference vegetation index (NDVI)

\begin{abstract}
:
As many studies which detect land cover changes using satellite imagery have been conducted previously; this study uses satellite imagery from Sentinel-2, which was launched by European Space Agency (ESA) in 2015. The main characteristics of Sentinel-2 are: a 10m spatial resolution in visible and Near-infrared (NIR) bands, a revisit frequency of 5 days based on combining Sentinel-2A and Sentinel-2B, and a free and open data policy. Using bands 4 and 8 of Sentinel2, NDVI is calculated to assess whether the target being observed contains live green vegetation. The difference was calculated by subtracting NDVI of one day from another. Changes from vegetation to built-up areas can be detected via the changes in NDVI. However, automatically computing land cover changes generates errors under present circumstances. In order to detect land cover change accurately, human review is required. This study focuses on how NDVI can assist analysts in quantifying land cover change. As a result of the analysis, land cover changes were extracted by differencing NDVI images of 2 periods, but some errors arose in the places where land cover did not change but NDVI fluctuated owing to other reasons. I show the land cover changes which were detected, the places where it is difficult to detect the change, and methods to reduce the errors.
\end{abstract}

